\title{
A computational protocol to fragment-based drug design at PDB
}

\section{scale}

\author{
F Moriaud ${ }^{* 1}$, T Henry ${ }^{1}$, SA Adcock ${ }^{1}$, AM Vorotynsev ${ }^{1}$, L Martin ${ }^{1}$, O Doppelt ${ }^{1}$, \\ AG De Brevern ${ }^{2}$ and F Delfaud ${ }^{1}$
}

Address: ${ }^{1}$ MEDIT SA, 2, rue du belvédère, 91120, Palaiseau, France and ${ }^{2}$ Paris, France

* Corresponding author

from 3rd German Conference on Chemoinformatics

Goslar, Germany. II-13 November 2007

Published: 26 March 2008

Chemistry Central Journal 2008, 2(Suppl I):S6 doi:I0.II86/I752-I53X-2-SI-S6

This abstract is available from: http://www.journal.chemistrycentral.com/content/2/SI/S6

(C) 2008 Moriaud et al.

Fragment- based drug design has emerged in the last decade. This exciting field has been recently reviewed [1]. Obtaining structural information on the fragment complexed to the protein target is a key factor and also a major limitation. Therefore, computational methods are needed to mine efficiently all the available 3D structures of ligands complexed to proteins, both treated as a whole and as smaller fragment to increase the likelihood of fragment hopping from one target to another.

MED-SuMo [2] is a target based drug design tool. This tool is based on the identification of local shape and 3D Surface Chemical Features similarities in the target binding site with other proteins (with their co-crystallized ligands). MED-SuMo uses the binding site of the target as a query to search either the whole PDB (or any corporate protein structure databank) for all the binding sites that display a local match with the query. This valuable information can then be used to identify which residues of the binding site are potentially important for affinity and selectivity. As the similar binding sites are overlaid, the cocrystallized ligands are aligned and are therefore a starting material for ligand hybridization. Among the hundreds of overlaid binding sites generated by MED-SuMo, we found the protein-ligand complexes overlaid by Pierce et al. [3] for ligand hybridization in the BREED method. Interestingly, they found relevant combinations of fragments starting from only a few protein-ligand complexes structures and we believe that the output of MED-SuMo is a very promising input for automatic methods like BREED.
In this work, we used MED-SuMo on a fragment database derived from the PDB: each pdb file is converted into one or more pdb files containing a single fragment as ligand. MED-SuMo is used to query and mine the Surface Chemical Functions surrounding the fragments, seeking similarities with the protein of interest. A library of fragments positioned in the active site and annotated with the counts of contacts and h-bonds is generated. This is the basis of a protocol which ranks and hybridizes fragments. Fragmentation and hybridization steps are coupled with substructure searches in a database of chemical compounds from suppliers to increase the likelihood of fragments and hybridized molecules as synthetically feasible molecules.

\section{References}

I. Hajduk PJ, Greer J: A decade of fragment-based drug design: strategic advances and lessons learned. Nat Rev Drug Discov 2007, 6(3):211-9.

2. Jambon M, Andrieu O, Combet C, Deléage G, Delfaud F, Geourjon C: The SuMo server : 3D search for protein functional sites. Bioinformatics 2005, 21:3929-3930.

3. Pierce AC, Bemis GW: BREED: generating novel inhibitors through hybridization of known ligands. Application to CDK2, p38, and HIV protease. J Med Chem 2004, 20:2768-75. 